\title{
Efficient Joint Unicast/Multicast Transmission over IEEE 802.11e WLANs
}

\author{
José Villalón, Pedro Cuenca and Luis Orozco-Barbosa
}

\author{
Albacete Research Institute of Informatics \\ Universidad de Castilla-La Mancha 02071 Albacete, Spain \\ \{josemvillalon,pcuenca,lorozco\}@dsi.uclm.es
}

\begin{abstract}
Multimedia communications over WLAN is widely acknowledged as one of the key, emerging applications for wireless LANs. As with any multiservice network, there is the need to provision the WLANs with the QoS mechanisms capable of guaranteeing the requirements of various services. The IEEE 802.11e (EDCA) standard defines the required QoS mechanisms into the protocol architecture of IEEE 802.11 WLANs. However, recent studies have shown that EDCA performs poorly when the medium is highly loaded due to the high collision rate. Numerous proposals have been reported in the literature aiming to overcome this main drawback. However, EDCA and the proposed mechanisms continue to have a serious problem with a specific type of application: the multicast traffic. In EDCA (as in DCF) the multicast service is defined as an unreliable service, i.e., it does not include the use of ACK frames. Furthermore, different to the unicast service, the multicast service makes use of a single rate out of the various rates included in the Basic Service Set (BSS) defined by the IEEE 802.11 standard. This situation has led many researchers to design techniques aiming to improve the multicast transmission such as Auto Rate Selection mechanism for Multicast (ARMS) and Hierarchical-ARSM (H-ARSM). In this paper we present a comparative performance evaluation when supporting different services, such as, voice, video, best-effort, background and in presence of multicast traffic. Our simulation results show the benefits present in multimedia applications when the unicast and multicast traffic are sent efficiently.
\end{abstract}

\section{Introduction}

A wide range of enterprise organizations have realized significant productivity increases by deploying mobile data applications using WLAN networks [1]. Motivated by the success, the organizations are now looking to further enhance the 
productivity of their mobile workers and leverage their WLAN network investments by deploying more applications into their networks. Multimedia communications is one of the leading applications that benefit from the mobility and increasing bit rates provided by current and emerging WLAN technologies. However, multimedia applications are not only characterized by their high bandwidth requirements, but also impose severe restrictions on delay, jitter and packet loss rates. In others words, multimedia applications require Quality of Service (QoS) support. Guaranteeing those QoS requirements in IEEE 802.11 is a very challenging task due to the QoS-unaware operation of its MAC layer. This layer uses the wireless media characterized by the difficulties faced by the signal propagation. Thus providing QoS to IEEE 802.11 has been and it is an active research area giving rise to numerous service differentiation schemes.

The IEEE 802.11e Working Group has worked hard on the specification of IEEE 802.11e standard [2]. The IEEE 802.11e defines the mechanisms for wireless LANs aiming to provide QoS support to time-sensitive applications, such as, voice and video communications. However, many studies have shown that the IEEE 802.11e (EDCA) scheme performs poorly under heavy load conditions. The severe degradation is mainly due to high collision rates. This reason has led many researchers to design new techniques aiming to address the shortcomings of the current standard.

Furthermore, EDCA and the proposed mechanisms to improve the QoS only have been designed to enhance the performance of unicast applications. In these schemes (as in DCF) the multicast service is defined as an unreliable service, i.e., it does not include the use of ACK frames. Furthermore, due the absence of a feedback, the multicast service makes use of a single rate out of the various rates included in the Basic Service Set (BSS) defined by the IEEE 802.11 standard. This situation has led many researchers to design techniques aiming to improve the multicast transmission.

In this paper, we address the two aforementioned issues by enhancing the multimedia communications over IEEE 802.11 WLANs. Our main objective has been to check the behavior of some QoS and multicast schemes proposed, in presence of uplink unicast traffic and downlink multicast traffic. The unicast traffic support different service, such as, voice, video, best-effort, background. For the downlink traffic, the access point transmits a video stream to the multicast receivers. The rest of the paper is organized as follows. Section 2 provides an overview of the IEEE 802.11 and the IEEE 802.11e WLAN standard, and some of the most relevant QoS proposals recently reported in the literature. In Section 3, we present an overview of some schemes to provide reliable multicast transmission in IEEE 802.11 WLAN. In Section 4, we carry out a comparative performance evaluation when supporting different unicast services, such as, voice, video, best-effort, background and in presence of multicast traffic. Finally, Section 5 concludes the paper. 


\section{The IEEE 802.11 DCF and QoS Enhancements}

The basic access function in IEEE 802.11 is the Distributed Coordination Function (DCF). A station operating under the DCF scheme should first sense the state of the channel before initiating a transmission. A station may start to transmit after having determined that the channel is idle during an interval of time longer than the Distributed InterFrame Space (DIFS). Otherwise, if the channel is sensed busy, once the transmission in course finishes and in order to avoid a potential collision with other active (waiting) stations, the station will wait a random interval of time (the Backoff_Time) before starting to transmit. As long as no activity is detected in the channel, a backoff counter, initially set to Backoff_Time, is decremented on an aSlotTime by aSlotTime basis. Whenever activity is detected, the backoff counter is frozen and reactivated once again when the channel has remained idle during an interval of time longer than DIFS. The station will be able to begin transmission as soon as the backoff counter reaches zero. In case of an unsuccessful transmission, the station will have a finite number of attempts, using a longer backoff time after each attempt.

Even though DCF is a simple and effective mechanism, DCF can neither support QoS nor guarantee to meet the multimedia applications requirements. It is for this reason that many researchers have proposed techniques the provisioning of QoS mechanisms into the DCF mode of operation. The description of such mechanisms is out of the scope of this work. An overview of many of the different QoS enhancements mechanisms for the IEEE 802.11 standards can be found in [3]. The need for a better access mechanism with an aim for providing service differentiation has led Task Group E of the IEEE 802.11 working group to come up with an extension to the IEEE 802.11 standard called IEEE 802.11e [2].

The IEEE 802.11e standard specifies the mechanisms enabling the provisioning of QoS guarantees in IEEE 802.11 WLANs. In the IEEE 802.11e standard, a third coordination function has been added: the Hybrid Coordination Function (HCF). HCF incorporates two new access mechanisms: the contention-based Enhanced Distributed Channel Access (EDCA) and the HCF Controlled Channel Access (HCCA).

EDCA has been designed to be used with the contention-based prioritized QoS support mechanisms. In EDCA, two main methods are introduced to support service differentiation. The first one is to use different IFS values for different ACs. The second method consists in allocating different CW sizes to the different ACs. Each AC forms an EDCA independent entity with its own queue and its own access mechanism based on an DCF-like mechanism with its own Arbitration Inter-

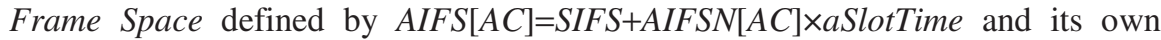
$\mathrm{CW}[\mathrm{AC}](C W \min [A C] \leq C W[A C] \leq C W \max [A C])$, where AIFSN[AC] is the Arbitration Inter Frame Space Number. If an internal collision arises among the queues within the same station, the one having higher priority obtains the right to transmit. The queue getting the right to access to the channel obtains a transmis- 
sion opportunity. The winning queue can then transmit during a time interval whose length is given by TXOPLimit.

Many on-going research efforts are focusing on the evaluation of the IEEE 802.11e standard [4]-[6]. Many studies have revealed that the poor performance exhibited by the standard is mainly due to the high collision rates encountered when a large number of stations attempt to access the channel. Numerous proposals have been reported in the literature aiming to overcome this main drawback. In the following, we undertake the analysis of three of the most prominent ones.

In this regard, the Fast Collision Resolution Mechanism FCR [7] aims to shorten the backoff period by increasing the contention window sizes of all active stations during the contention resolution period. To reduce the number of wasted (idle) slots, the FCR algorithm assigns the shortest window size and idle backoff timer to the station having successfully transmitted a packet. Moreover, when a station detects a number of idle slots (static backoff threshold), it starts reducing the backoff timer exponentially, instead of linearly as specified by the EDCA standard. To address the provisioning of QoS mechanisms, the authors further introduce an enhanced version of the FCR algorithm, namely, the Real Time Fast Collision Resolution (RT-FCR) [7] algorithm. In this algorithm, the priorities are implemented by assigning different backoff ranges based on the type of traffic. In their study, the authors have considered three main traffic types: voice, video, and best-effort (data) traffic.

Under this scheme, voice packets hold the highest priority to access the channel by setting $\mathrm{CW}=\mathrm{CW}_{\min }$. All the other flows have to wait, at least, eight backoff slots before being allowed to gain access to the channel. The video traffic is assigned the second highest priority by using a smaller maximum $\mathrm{CW}$ size than the one assigned to the best-effort data traffic.

In [8], Mali et al. go a step further by introducing a new scheme called Adaptive Fair EDCF (AFEDCF). This mechanism uses an adaptive fast collision resolution mechanism (similar to the FCR mechanism) when the channel is sensed idle. In contrast with the FCR mechanism, AFEDCF computes an adaptive backoff threshold for each priority level by taking into account the channel load.

However, the main deficiency of these mechanisms comes from its inability to provide the proper QoS to the video service in scenarios comprising legacy DCFbased and IEEE 802.11e stations. This is due to the fact that, under theses schemes, the video packets have always to wait for a minimum of eight backoff slots in order to comply with the highest priority assigned to the voice traffic. Under these schemes, the presence of voice and DCF stations may even result in starvation to the video flows. Moreover, the implementation of these mechanisms implies incompatible modifications to the IEEE 802.11e specifications.

Based on limitations of these mechanisms, in [9] we proposed a new IEEE802.11e (called B-EDCA) based QoS mechanism compatible with the IEEE 802.11e specifications and capable of providing QoS support, particularly to video applications. Based on the results obtained in one of our previous studies we found out that the IFS (denoted AIFS in the EDCA standard) is the most important and 
critical parameter enabling the provisioning of QoS to multimedia applications. Our proposal has been based in using the minimum waiting time necessary to continue decrementing the backoff counter of the multimedia flows.

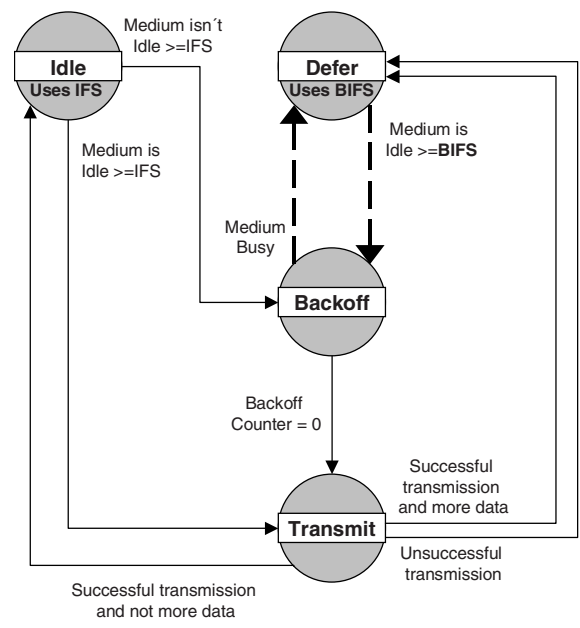

Fig. 1 B-EDCA Proposed Mechanism.

In order to introduce our proposal, we toke a closer look at the mode of operation of the DCF and EDCA schemes, and particularly on the role played by the IFS (AIFS) parameter. The IFS (AIFS) is used in the idle and the backoff states (see fig. 1). According to the current DCF and EDCA standards, the same values for the IFS parameter should be used regardless of the state in which the station is. In B-EDCA we propose to use a different set of IFS values depending on the state in which the station is. We have however to ensure not to compromise the operation of the HCF. We then propose the following parameter setting:

1. In the Idle state. The stations will use the IFS values as specified in the IEEE 802.11e standard including the Hybrid Coordination Function. This also ensures compatibility with the IEEE 802.11 (DCF) mechanism.

2. In every transfer from the Defer state to the Backoff state, we propose to use a different parameter, equivalent to the IFS, denoted from now on by BIFS. We then propose setting up this parameter to one, i.e., BIFS $=1$, for the voice and video services. In this way, we improve considerably the performance of voice and video applications, increasing their priorities with respect to other flows. This setting also ensures that the $\mathrm{HC}$ will keep the highest priority. According to this mechanism, the stations must wait at least one additional slot during the backoff procedure before being allowed to transmit since the backoff interval is set within the $[1, \mathrm{CW}+1]$ range. We then propose using the set of values for BIFS to 1-1-3-7 for voice, video, best-effort and background traffics, respectively. 


\section{Multicast transmission over IEEE 802.11}

Multicasting in IEEE 802.11 WLAN is specified as a simple broadcasting mechanism that does not make use of ACK frames. There are two main issues to be addressed to effectively deploy reliable and scalable multicast services over IEEE 802.11 wireless LANs. First, the absence of a feedback mechanism has a strong impact on the reliability of the service provided to the user. Second, according to the IEEE $802.11 \mathrm{a} / \mathrm{b} / \mathrm{g}$ standards, all frames with multicast and broadcast Receiver Address (RA) should be transmitted at one of the rates included in the basic rate set.

Most research efforts on multicasting in IEEE 802.11 WLANs have focused on improving the service reliability by integrating ARQ mechanisms into the protocol architecture. In [10], the Leader-Based Protocol (LBP) ARQ mechanism has been introduced to provide the multicast service with some level of reliability. To address the ACK implosion problem, LBP assigns the role of group leader to the multicast receiver exhibiting the worst signal quality in the group. The group leader holds the responsibility to acknowledge the multicast packets on behalf of all the multicast group members, whereas other MTs may issue Negative Acknowledgement (NACK) frames when they detect errors in the transmission process.

Recently, the Rate Adaptive Multicast (RAM) scheme has been proposed in [11] for reliable multicast delivery. Similar to the LBP scheme, the transmitter has first to send a RTS frame to indicate the beginning of a multicast transmission. However, in RAM the RTS frame is used by all the multicast receivers to measure the Receiver Signal Strength (RSS). Then, each multicast receiver has to send a variable length dummy CTS frame whose length depends on the selected PHY transmission mode. Finally, the transmitter senses the channel to measure the collision duration and can adapt the PHY rate transmission of the multicast data frame accordingly. Thus, RAM adapts the PHY transmission rate of the multicast data frames to the channel conditions. However, the overhead introduce by this mechanism is very high. In fact, the RTS/CTS option is disabled in most 802.11 products currently available in the market.

For reduce this overhead, we presented in [12] a novel mechanism named Auto Rate Selection mechanism for Multicast (ARSM) in multirate wireless LAN. Basically, the ARSM mechanism dynamically selects the multicast data rate based on the channel conditions perceived by the MTs. The main idea behind our proposal is to identify the AP to MT channels exhibiting the worst conditions, expressed in terms of the SNR ratio. The AP assigns the role of group leader to this MT. The group leader holds the responsibility of acknowledging the multicast packets on behalf of all the multicast group members, whereas other MTs may issue NACK frames when they detect errors in the transmission process, in that case the AP retransmit the frame. The mechanism to discover this station is named Multicast Channel Probe Operation (MCPO). In order to reduce the amount of processing 
to be carried out by the MTs, we proposed a Dynamic Multicast Data Transmission procedure by making use of several multicast PHY data rates. Under this scheme, the AP can be found in one of two different states depending on the feedback received.

1. While the AP successfully delivers multicast data frame, the MCPO is deactivated. In this state, the AP will adapt its PHY data rate using the SNR value contained in the received ACK coming from the group leader.

2. If the AP shows a failure of $N_{t h}$ consecutive multicast transmissions (detected via NACKs), it initiates the MCPO.

Even though ARSM has proved to be effective as reported in [12], such setting heavily penalizes those MTs exhibiting better channel conditions. For this reason, we presented in [13] an enhancing the ARSM scheme to address the aforementioned issues. This second mechanism is named Hierarchical ARSM (H-ARSM). In this scheme, we focus our attention to its use in multicast video services. Under H-ARSM, the video is expected to be encoded into two layers, namely the base and enhancement layers. The packets containing the base layer of the video are sent to all the members of the multicast group following the rules as established by ARSM. In this way, the mechanism should guarantee a minimum video quality to all users. In the case of the enhancement layer, the operating mode is quite similar to the one used by the ARSM scheme, i.e., the AP has to first select a group leader for the enhancement layer, whose main mission is to acknowledge (ACK) the packets sent to the group. The other MTs may issue NACK frames when they detect errors in the transmission process, in that case the AP retransmit the frame. The main difference for enhancement layer comes from the fact that instead of selecting one of the members with the lowest SNR as the group leader, this new mechanism selects one of the members with the highest SNR. Furthermore, the enhancement layer is sent at a rate no lower than $5.5 \mathrm{Mbps}$

\section{Performance Evaluation}

In this section, we carry out a performance analysis of our proposed mechanism. Our main objective is show the performance of our proposed schemes for QoS and multicast transmission (B-EDCA, ARSM and H-ARSM) in presence of multicast traffic and different unicast applications. We compare the performance of EDCA with the compatible B-EDCA mechanism as access model to the channel. Concern to the multicast mechanism, in [12] we showed that the standard is unable to effectively provide multicast services, and ARSM enhance the performance of LBP and RAM mechanism. For this reason, we study ARSM and H-ARSM as method to send the multicast traffic. Table I shows the different combinations studied in this paper. 
Table 1. Combinations used to access the channel and for the multicast traffic.

\begin{tabular}{ccc}
\hline \hline & Access Model & Multicast scheme \\
\hline ARSM-EDCA & EDCA & ARSM \\
ARSM_BEDCA & B-EDCA & ARSM \\
HARSM-EDCA & EDCA & H-ARSM \\
HARSM-BEDCA & B-EDCA & H-ARSM \\
\hline
\end{tabular}

Throughout our study, we have made use of the OPNET Modeler tool 11.5 [14], which already integrates the IEEE 802.11 simulator. We have integrated into it the EDCA, B-EDCA, ARSM and H-ARSM mechanisms.

In our simulations, we model an IEEE 802.11b WLAN consisting of an AP, nine multicast wireless MTs receivers, and eight unicast wireless MTs. We have varied the network size expressed in terms of the area covered by the AP and multicast MTs. The network size has been initially set to a geographical area of $50 \mathrm{~m} \mathrm{x}$ $50 \mathrm{~m}$. We have then increased the network size in both dimensions by $10 \mathrm{~m} \times 10 \mathrm{~m}$ to a maximum network size of $140 \mathrm{~m} \times 140 \mathrm{~m}$. The access point is located in the center of the BSS. The cell size is changed throughout the different scenarios under study. The multicast MTs move randomly within the BSS at a constant speed of $5 \mathrm{~km} / \mathrm{h}$, whereas the unicast MTs are static and placed close to the access point. We assume that the unicast packets are always transmitted at $11 \mathrm{Mbps}$.

In our scenarios, we have assumed the use of two types of traffic flows: downlink multicast traffic and uplink unicast traffic. For the downlink traffic, the access point transmits a video stream to the multicast MTs. For the video streaming source, we have used traces generated from a variable bit-rate H.264 video encoder [15]. We generate two types of traces corresponding to the use or not of the hierarchical video coding scheme presented in [13]. We have used the sequence Mobile Calendar encoded on CIF format at a video frame rate of 25 frames/s. When the hierarchical coding is used, the base layer accounts approximately for $30 \%$ of the total video data $(209 \mathrm{Kbps})$. Throughout our experiments, we have confirmed that an acceptable video quality in the base layer can be obtained while the amount of data traffic pertaining to the enhancement layer is in the order of 70 $\%$ (489 Kbps). The average video transmission rates used has been around 650 Kbps and $700 \mathrm{Kbps}$ corresponding to the use or not of the hierarchical video coding.

For the unicast traffic our scenarios have eight unicast stations, with the following ratio: 2 voice, 2 video, $2 \mathrm{BE}$ and $2 \mathrm{BK}$ stations. We assume the use of constant bit-rate voice sources encoded at a rate of $16 \mathrm{kbits} / \mathrm{s}$ according to the $\mathrm{G} .728$ standard [16]. The voice packet size has been set to 168 bytes including the RTP/UDP/IP headers. For the video applications, we have made use of the traces generated from a variable bit-rate H.264 video encoder. We have used the sequence mobile calendar encoded on CIF format at a video frame rate of 25 frames/sec. The average video transmission rate is around $480 \mathrm{kbits} / \mathrm{s}$ with a 
packet size equal to 1064 bytes (including RTP/UDP/IP headers). The best-effort and background traffics have been created using a Pareto distribution traffic model. The average sending rate of is $128 \mathrm{kbit} / \mathrm{s}$, using a 552 bytes packet size (including TCP/IP headers). All traffic sources are randomly activated within of the interval $[1,1.5]$ seconds from the start of the simulation. Throughout our study, we have simulated two minutes of operation of each particular scenario.

In our study, we have been interested in assessing the performance in terms of the following metrics: Normalized throughput, packet loss rate and video quality. To be able to compare the results at different loads (traffic patterns of different applications), we have preferred plotting the normalized throughput rather than the absolute throughput. The normalized throughput is calculated as the percentage of the offered load actually delivered to destination.

In order to limit the delay experienced by the unicast video and voice applications, the maximum time that video packet and voice packet may remain in the transmission buffer has been set to $100 \mathrm{~ms}$ and $10 \mathrm{~ms}$, respectively. For the multicast video traffic (video streaming), we are selected $2 \mathrm{~s}$. These time limits are in line with the values specified by the standards and in the literature. Whenever a video or voice packet exceeds these upper bounds, it is dropped. The loss rate due to this mechanism is given by the packet loss rate due to deadline.

Finally, one of the most important metrics in multimedia communications is the quality of the received signal as perceived by the end user. This has been evaluated using the Video Quality Metric (VQM) [17] for the video signal. These metrics have been proved to behave consistently with the human judgments according to the quality scale that is often used for subjective testing (MOS, Mean Opinion Score) in the engineering community (see Table II).

Table 2. Quality scale (MOS)

\begin{tabular}{rcc}
\hline \hline Rating & Impairment & Quality \\
\hline 5 & Imperceptible & Excellent \\
4 & Perceptible, not annoying & Good \\
3 & Slightly & Fair \\
2 & Annoying & Poor \\
1 & Very annoying & Bad \\
\hline
\end{tabular}

Fig. 2 shows the normalized throughput obtained for the Vo, Vi, BE and BK services and the global throughput when making use of each one of combinations being considered. From the results, it is clear that H-ARSM offers better results than the ARSM scheme. When H-ARSM is used to send the multicast traffic, the unicast normalized throughput for all the traffic is 100\%. In H-ARSM, the base layer is sent at low rates (the same rate used in ARSM) while the enhancement layer is sent at a rate no lower than 5.5Mbps. For this reason, H-ARSM need less time to send the multicast video streaming, and the other applications can to send 


\section{Wireless and Mobile Networking}

more data packets. Figure also shows that the B-EDCA mechanism outperforms the EDCA mechanism when the multicast traffic is sent to ARSM. B-EDCA use the minimum waiting time necessary to continue decrementing the backoff counter of the voice and video flows. B-EDCA introduces a new reserved slot to the voice and video traffic, and reduces the collision present between these applications and the other traffic.

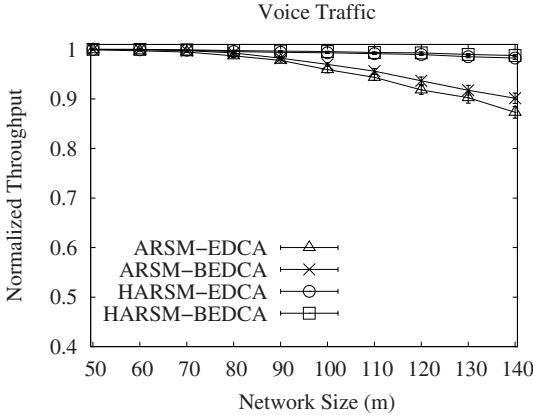

(a)

BE Traffic

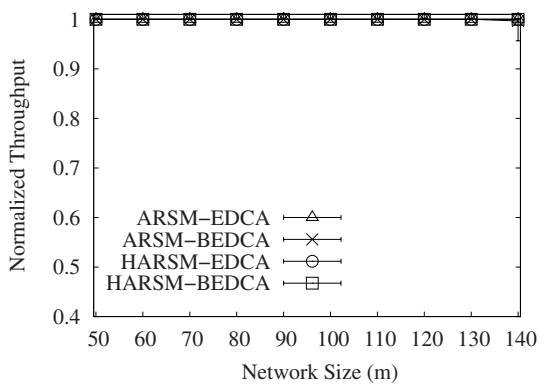

(c)

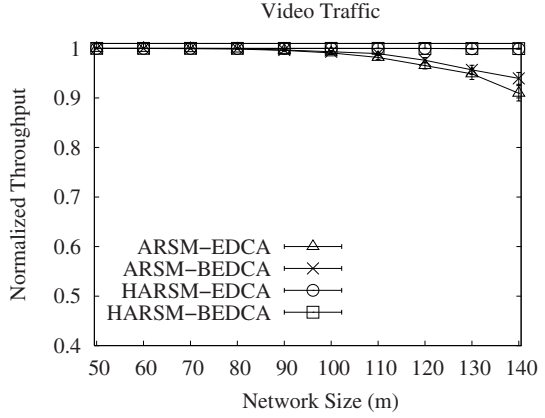

(b)

BK Traffic

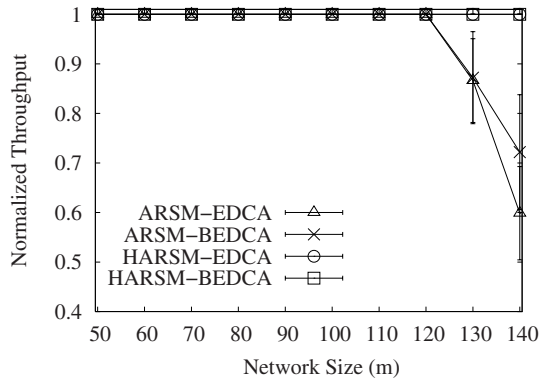

(d)

Global Throughput

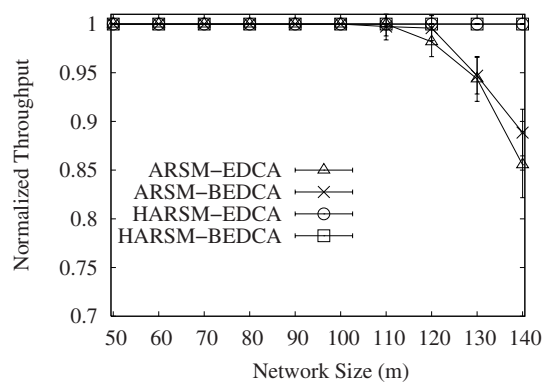

(e)

Fig. 2 Unicast Normalized Throughput: a) Voice, b) Video, c) Best-Effort d) Background and e) Total Traffic. 
In the case of the multicast traffic, in Figure 3.a it is clear that the H-ARSM scheme is capable of sending all the traffic associated to the base layer $(\mathrm{H}$ ARSM.HP ${ }^{1}$ ) independently of the network size. However, the throughput of the enhancement layer (H-ARSM.LP) decreases as the network size is increased. The reduction experienced by the enhancement layer is due to the decrease of the MTs pertaining to the enhancement layer as the network size increase. Figure 3.b shows the packet loss rate for the different scenarios. In the case of H-ARSM, the only packets being lost belong to those carrying the enhancement layer and being addressed to the stations not belonging to the enhancement multicast group. The $\mathrm{H}$ ARSM scheme guarantees the correct delivery of all the packets containing the base layer to all the members of the multicast group (H-ARSM.HP). The scheme also guarantees the correct delivery of all the packets of the enhancement layer to all the members of the enhancement group. In the case of the ARSM and scheme, all the packet losses are due to excessive delay by the packets while waiting to be transmitted. H-ARSM limits the packet losses (belongs to enhancement layer) to those stations exhibiting the worst channel conditions, i.e., those stations belonging only to the base layer multicast group. With ARSM, all the members of the multicast group are affected.

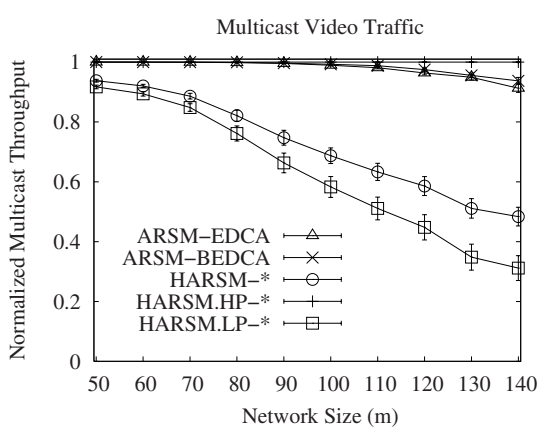

(a)

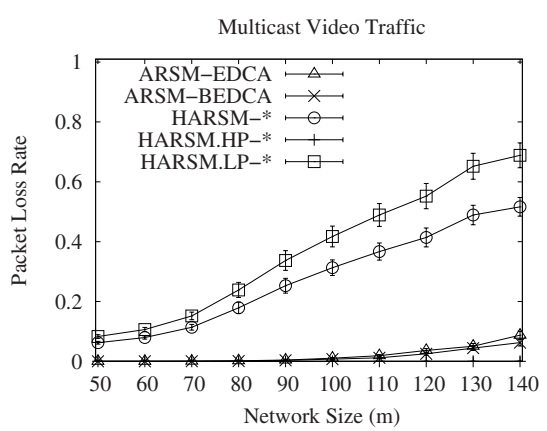

(b)

Fig. 3 Multicast Traffic: a) Normalized Throughput and b) Multicast Packet Loss Rate.

Finally, Figure 4 shows the video quality for unicast and multicast video traffic. The figure 4.a shows that when H-ARSM is used to send de multicast video streaming, the quality of the unicast video received is not dependent on network size. However, when ARSM is used, this quality decrease when the network size increase. In the case of the multicast traffic, when ARSM mechanism is used, the figure 4.b shows a video quality around 4.2 (corresponding a quantization factor equal to 26) as long as no packet losses are reported. However, the video quality drastically falls as the packet loss rate increases (network sizes beyond $90 \mathrm{~m}$ ) as a

\footnotetext{
${ }^{1}$ HARSM-*, HARSM.HP-* and HARSM-LP-* symbolize the H-ARSM, H-ARSM base layer and H-ARSM enhanced layer respectively used whit EDCA and B-EDCA mechanism
} 
result of missing the packet delivery deadlines. In the case of the H-ARSM, a minimum video quality is always guaranteed since the base layer is always delivered.

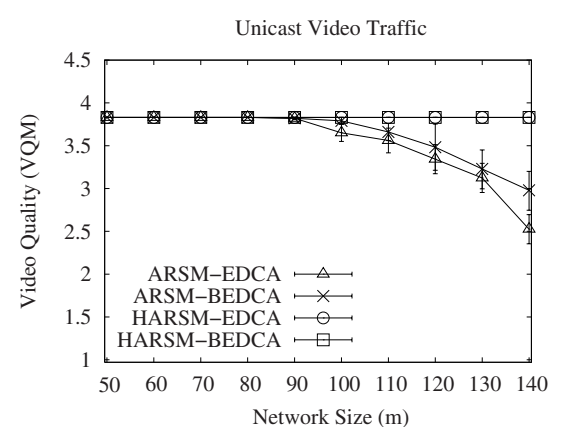

(a)

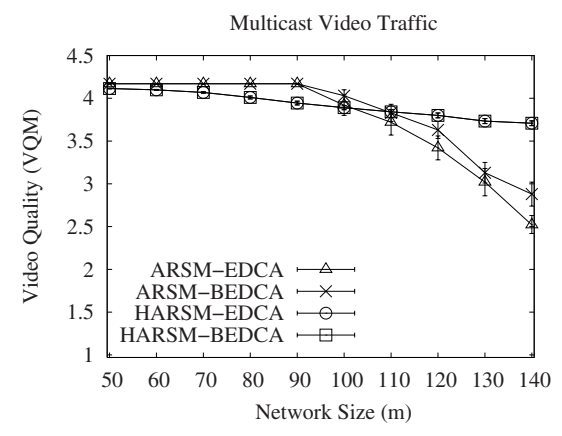

(b)

Fig. 4 Video Quality (VQM): a) Unicast Video and b) Multicast Video.

\section{Conclusions}

In the first part of this paper, we have overviewed some of the most relevant works in the area of QoS provisioning and reliable multicast for IEEE802.11 wireless LANs. Our main objective has been to show the performance of our proposed schemes for QoS and multicast transmission (B-EDCA, ARSM and H-ARSM) in presence of multicast traffic and different unicast applications. Our results obtained have shown that B-EDCA mechanism outperforms the EDCA mechanism. Our results also have shown that H-ARSM carry out efficient sending for the multicast traffic, maximizing the available resources for unicast traffic. The results obtained have shown that H-ARSM outperforms the ARSM mechanism for unicast and multicast traffic.

\section{Acknowledgments}

This work was supported by the Ministry of Science and Technology of Spain under CONSOLIDER CSD2006-46 and CICYT TIN2006-15516-C04-02, the Council of Science and Technology of Castilla-La Mancha under project PAI06-0106 and FEDER 


\section{References}

1. LAN MAN Standards Committee of the IEEE Computer Society, ANSI/IEEE Std 802.11, "Part 11: Wireless LAN Medium Access Control (MAC) and Physical Layer (PHY) Specifications", 1999 Edition.

2. IEEE 802 Committee of the IEEE Computer Society, ANSI/IEEE Std 802.11e, "Part 11: Wireless LAN Medium Access Control (MAC) and Physical Layer (PHY) Specifications: Medium Access Control (MAC) Quality of Service (QoS) Enhancements", Nov. 2005.

3. F. Mico, P. Cuenca and L.Orozco Barbosa "QoS Mechanisms for IEEE 802.11 Wireless LANs". Lecture Notes in Computer Science. Vol. 3079. pp. 609-623, 2004.

4. D. Vassis and G. Kormentzas, "Delay Performance Analysis and Evaluation of IEEE 802.11e EDCA in Finite Load Conditions". Wireless Personal Communicat., vol. 34. pp. 29-43. 2005.

5. Z.K.Tsang, D.H.K. Bensaou and B. D. Gao, "Performance Analysis of IEEE 802.11e Contention-based Channel Access". IEEE Journal on Selected Areas in Communications. vol. 22 .pp. 2095-2106. 2004.

6. Q.Ni, "Performance Analysis and Enhancements for IEEE 802.11e Wireless Networks". IEEE Network. vol.19. no.4. pp. 21-26. 2005.

7. Y. Kwon, Y. Fang and H. Latchman, "Design of MAC Protocols with Fast Collision Resolution for Wireless Local Area Networks". IEEE Trans. on Wireless Communicat., vol. 3, no.3. pp. 793-807, May 2004.

8. M. Malli, Q. Ni, T. Turletti and C. Barakat "Adaptive Fair Channel Allocation for QoS Enhancement in IEEE 802.11 Wireless LANs", in Proceedings of IEEE ICC, Paris, Jun. 2004.

9. J. Villalón, P. Cuenca and L. Orozco-Barbosa "B-EDCA: A New IEEE 802.11e-based QoS Protocol for Multimedia Wireless Communications" Lecture Notes in Computer Science. Vol. 3976. pp. 6148-159, 2006.

10. Joy Kuri and Sneha Kumar Kasera, "Reliable Multicast in Multi-access Wireless LANs", ACM Wireless Networks, Volume 7, Issue 4, Pages 359 - 369, 2001.

11. A. Basalamah, H. Sugimoto and T. Sato "Rate Adaptive Reliable Multicast MAC Protocol for WLANs". Proc. of VTC, Melbourne, Australia, May 2006. pp 1216-1220.

12. J. Villalón, Y. Seok, T. Turletti, P. Cuenca and L. Orozco-Barbosa, "ARSM: A Cross-Layer Auto Rate Selection Multicast Mechanism for Multi-Rate Wireless LANs". IET Communications, Vol. 1-5. pp. 893-902. October 2007.

13. J. Villalón, Y. Seok, T. Turletti, P. Cuenca and L. Orozco-Barbosa, "Cross-Layer Architecture for Adaptive Video Multicast Streaming over Multirate Wireless LANs". IEEE Journal on Selected Areas in Communications, Vol. 25-4. pp. 699-711, May 2007.

14. Opnet.Technologies.Inc. OPNET Modeler 11.5 (c)1987-2006. http://www.opnet.com.

15. ITU-T Recommendation H.264, "Advanced Video Coding For Generic Audiovisual Services". May 2003.

16. ITU-T Recommendation G.728, "Coding of Speech at $16 \mathrm{kbit} / \mathrm{s}$ using Low-Delay Code Excited Linear Prediction", Std., September 1992.

17. S. Wolf and M. H. Pinson, "Video Quality Measurement Techniques". NTIA Report 02-392. June 2002. 